\title{
Profiling and Integrated Analysis of Differentially Expressed Circular RNAs in Plasma Exosomes as Novel Biomarkers for Advanced-Stage Lung Adenocarcinoma
} OncoTargets and Therapy

\author{
Shan Lin ${ }^{1,2, *}$ \\ Wenji Xiong ${ }^{3, *}$ \\ Huibo $\mathrm{Liu}^{4}$ \\ Liping Pei ${ }^{5}$ \\ Huanfa $Y_{i}{ }^{1}$ \\ Yinghui Guan ${ }^{2}$ \\ 'Central Laboratory, The First Hospital \\ of Jilin University, Changchun, Jilin, \\ People's Republic of China; ${ }^{2}$ Department \\ of Respiratory Medicine, The First \\ Hospital of Jilin University, Changchun, \\ Jilin, People's Republic of China; \\ ${ }^{3}$ Department of Radiology, The First \\ Hospital of Jilin University, Changchun, \\ jilin, People's Republic of China; \\ ${ }^{4}$ Department of Dermatology and \\ Venerology, The First Hospital of Jilin \\ University, Changchun, Jilin, People's \\ Republic of China; ${ }^{5}$ Department of \\ Echocardiography, The First Hospital of \\ Jilin University, Changchun, Jilin, People's \\ Republic of China \\ *These authors contributed equally to \\ this work
}

Purpose: Exosomes contain abundant circRNAs and are determined to be involved in the pathogenesis of lung adenocarcinoma (LUAD). Thus, our study aimed to explore new circRNAs in plasma exosomes that could be involved in such pathogenesis.

Patients and Methods: High-throughput sequencing was used in identifying the alterations in exosomal circRNA expression. Gene ontology functional analysis (GO) and Kyoto Encyclopedia of Genes and Genomes (KEGG) pathway analysis were performed to determine the significant functions and pathways associated with differentially expressed circRNAs. TargetScan and miRanda were used to predict circRNA-targeted microRNAs and mRNAs. CircRNA expression profiles were then validated by quantitative reversetranscription polymerase chain reaction (qRT-PCR). Wound healing and Transwell assays were performed to determine the roles of has_circ_0102537 in LUAD progression.

Results: We identified six significantly upregulated and 214 significantly downregulated circRNAs. GO and KEGG pathway analysis suggested that the differentially expressed circRNAs are involved in the occurrence and development of LUAD. A circRNAmiRNA-mRNA meshwork was established to predict the potential interactions among these RNAs. The circRNA expression profile was then subjected to qRT-PCR for validation. We identified hsa_circ_0102537 to be downregulated in both LUAD plasma exosomes and tissues. GO, KEGG pathway analysis, circRNA-miRNA-mRNA meshwork, and further experiments suggest that hsa_circ_0102537 could be involved in LUAD progression.

Conclusion: Our study explored a large number of circRNAs that may be involved in the LUAD pathogenesis, thereby supporting the need for further research on both diagnosis biomarkers and the potential intervention therapeutic targets.

Keywords: advanced-stage lung adenocarcinoma, plasma, exosomes, circular RNA, microRNA

\section{Introduction}

Lung cancer has been identified as the leading cause of cancer incidence and mortality, with 2.1 million new cases and 1.8 million deaths predicted worldwide in 2018. ${ }^{1}$ Lung cancer is mainly divided into small cell lung cancer (approximately $15 \%$ ) and non-small cell lung cancer (NSCLC, about 85\%). The main histologic subtypes of NSCLC include lung adenocarcinoma (LUAD) and lung squamous cell carcinoma. The incidence of LUAD has been observed to be increasing, year
Yinghui Guan

Department of Respiratory Medicine, The First Hospital of Jilin University, No. I

Xinmin Street, Changchun, Jilin I3002I, People's Republic of China

Email yinghuig20I6vip@163.com 
after year, eventually becoming as the most common subtype of NSCLC. ${ }^{2}$ Despite advances in early diagnosis and multimodality therapy, most LUAD patients have locally advanced or metastatic disease at the time of diagnosis, and the 5-y survival rate of $15 \%$ has not been improved. ${ }^{3}$ Reducing the morbidity and mortality of LUAD is currently an essential topic in medicine and healthcare research. Late diagnosis and delayed treatment have been determined to be the major obstacles for improving low survival rates and avoiding costly treatments; thus, it is critical to identify rapid and accurate molecular markers for early diagnosis. Moreover, additional research is needed in order to determine the molecular mechanism of LUAD and further explore novel therapeutic targets for advanced-stage LUAD.

Exosomes have been identified as lipid bilayerenclosed vesicles, smaller than $200 \mathrm{~nm}$ in diameter, and are secreted by all cell types. Exosomes exist in multifarious body fluids, including blood, urine, saliva, bronchoalveolar lavage fluid, follicle fluid, and amniotic fluid. ${ }^{4}$ Exosomes are composed of proteins, lipids, glycoconjugates, and nucleic acids. Exosomes contain a number of soluble proteins in the lumen that are found to be associated with the membranes, including integral membrane proteins, lipid-anchored membrane proteins, and peripherally associated membrane proteins. ${ }^{5}$ In general, a broad array of proteins can be used as exosomal positive markers, such as tetraspanin proteins (CD9, CD63, CD81, and CD82), heat shock proteins (Hsp70 and Hsp90), exosomal scaffolding proteins (alix and TSG101), membrane transport and fusion proteins (GTPases, annexins, and flotillin), and some negative proteins (GM130 and GRP78). ${ }^{6}$ Exosomes have been determined to be abundant in noncoding RNAs (ncRNAs), including microRNAs (miRNAs), circRNAs, transfer RNAs, etc. ${ }^{7,8}$ Proteins and nucleic acids in exosomes serve as cargo for signal transduction and intercellular communication. ${ }^{5}$ Recent studies have intensively focused on the roles of exosomes in tumorigenesis and tumor progression, such as in patients with lung cancer. Exosomes contribute to multiple pathological processes of lung cancer, including carcinogenesis, angiogenesis, the epithelial-mesenchymal transition (EMT), and metastasis. ${ }^{9}$ Exosomes released from lung cancer cells contain abundant TGF- $\beta$ and IL-10, which further induce cancer cell proliferation and invasion. ${ }^{10}$ Snaill found in the exosomes derived from cancerassociated fibroblasts promotes the EMT in lung cancer cells. ${ }^{11}$ Exosomal lncRNA H19 targets chemotherapy- sensitive NSCLC cells and confers gefitinib resistance. ${ }^{12}$ These results have led to the hypothesis that exosomes may represent a novel diagnostic marker and therapeutic target for LUAD.

Circular RNAs (circRNAs) are endogenous noncoding RNAs that form covalently-closed molecules with continuous loops without the $5^{\prime}$ caps or $3^{\prime}$ poly (A) tails that protect them from degradation by RNase R. ${ }^{13}$ Most circRNAs are comprised of exons, though others are comprised of either introns only or exons and introns. ${ }^{14}$ Widely expressed CircRNAs are highly conserved and diverse ${ }^{15}$ and are expressed in a tissue-specific and spatiotemporally specific manner. ${ }^{16}$

Originally, circRNAs were regarded as nonfunctional "junk" RNAs; however, they were recently demonstrated to possess multiple biological functions. Moreover, circRNAs modulate transcription, splicing, sponge microRNAs (miRNAs), interact with proteins, and serve as templates for polypeptide synthesis. ${ }^{17} \mathrm{~A}$ significant characteristic of circRNAs is their ability to adsorb miRNAs and block their binding to noncoding regions of mRNAs to subsequently reverse their inhibitory actions on target-gene transcription. ${ }^{14}$

Recent studies show that circRNAs are abundant in exosomes, indicating their potential use for diagnosis and monitoring of pathologies such as polycystic ovary syndrome, ${ }^{18}$ cardiovascular disease, ${ }^{19}$ traumatic brain injury, ${ }^{20}$ and cancers. $^{21}$ For example, in patients with osimertinibresistant NSCLC, the level of circ_0002130 in serum exosomes is upregulated. Further, circ_0002130 regulates the osimertinib resistance of NSCLC cells through targeting miR-498. ${ }^{22}$ In LUAD cancer cells, circRNA_0002178 enhances PD-L1 expression by sponging miR-34. Further, circRNA_0002178 can be delivered via exosomes into T-cells to induce cell exhaustion by upregulating PD-1 expression. ${ }^{23}$ Moreover, circSATB2 is highly expressed in tissues and serum exosomes from patients with lung cancer and is transferred by exosomes to promote the proliferation and invasion of NSCLC cells. ${ }^{24}$ Therefore, we hypothesized that exosomal circRNAs may play a key role in regulating signaling pathways and gene expression in LUAD, ultimately affecting pathogenesis.

Therefore, we explored novel circRNAs by examining circRNA expression in serumal exosomes isolated from advanced-stage LUAD patients by using high-throughput whole transcriptome sequencing. Additionally, functions of differentially expressed circRNAs were predicted by gene ontology (GO) and Kyoto Encyclopedia of Genes 
and Genomes (KEGG) pathway analyses. We then dissected the characteristics of key differential circRNAs and explored interactions among the different types of RNAs by constructing a circRNA-miRNA-mRNA network. Five randomly selected circRNAs were used to confirm the reliability of RNA sequencing results through quantitative reverse-transcription polymerase chain reaction (qRT-PCR). We profiled tissue circRNAs in LUAD tissues and paracancerous normal tissues using the Gene Expression Omnibus (GEO) database and found hsa circ_0102537 was downregulated in our RNA-seq and GSE101586. We found that circ_0102537 inhibited the migration and invasion of H1299 cells in vitro.

Collectively, our study revealed that the differentially expressed exosomal circRNAs might contribute to the initiation and progression of LUAD. Our study also underscored the potential importance of hsa_circ_0102537, which may serve as a biomarker for LUAD diagnosis.

\section{Patients and Methods}

\section{Patients}

For this study, 13 advanced-stage LUAD patients were recruited from The First Hospital of Jilin University (Changchun, China), whereas 12 healthy people were included as study controls (Table 1). Key exclusion criteria were as follows: having other malignant tumors, severe liver and kidney disease, hypertension, ischemic heart disease, and diabetes or other systemic diseases and being pregnant. In total, 13 plasma samples were collected from advancedstage LUAD patients who did not receive surgical resection, chemotherapy, or radiotherapy. For circRNA sequencing

Table I Clinical Characteristics of the Patients

\begin{tabular}{|l|l|l|l|l|}
\hline & Age & Gender & TNM Stage & Sample Use \\
\hline Case 1 & 64 & male & T4N2Mx & RNA-seq and qRT-PCR \\
Case 2 & 70 & female & T4N2MI & RNA-seq and qRT-PCR \\
Case 3 & 48 & female & T4N3MI & RNA-seq and qRT-PCR \\
Case 4 & 67 & male & T4N2MI & RNA-seq and qRT-PCR \\
Case 5 & 55 & female & T4N2MI & RNA-seq and qRT-PCR \\
Case 6 & 75 & female & T3N3MI & qRT-PCR \\
Case 7 & 69 & male & T4N3MI & qRT-PCR \\
Case 8 & 60 & male & T3N3MI & qRT-PCR \\
Case 9 & 59 & male & T2N2Mx & qRT-PCR \\
Case 10 & 70 & female & T3N3MI & qRT-PCR \\
Case 1I & 70 & male & T4N3Mx & qRT-PCR \\
Case 12 & 79 & female & T2N3MI & qRT-PCR \\
Case 13 & 70 & female & T4N3MI & qRT-PCR \\
\hline
\end{tabular}

Abbreviation: qRT-PCR, quantitative reverse-transcription polymerase chain reaction. analysis, plasma samples from five LUAD and five healthy subjects were selected. These samples were also used in verifying the accuracy of the sequencing results. All plasma samples from 13 LUAD and 12 healthy control subjects were used for further qRT-PCR validation experiments. This study was approved by the Ethics Committee of The First Hospital of Jilin University (clinical trial registration number was 2019-264) and adhered to the Declaration of Helsinki. Written informed consent was obtained from each participant.

\section{Isolation and Characterization of Exosomes}

Venous blood samples were collected in EDTA tubes. The plasma was immediately isolated using a horizontal rotor centrifugation $(3000 \times \mathrm{g}, 15 \mathrm{~min})$ at $4{ }^{\circ} \mathrm{C}$ and then filtered (Sartorius Minisart NML). Exosomes were isolated using an exoEasy Maxi Kit (Qiagen), following the manufacturer's procedure. Exosomes were identified using the transmission electron microscopy (TEM) and Western blot analysis. Exosomes were then fixed in $2 \%$ osmic acid solution, dehydrated, embedded, and stained with uranium acetate and lead acetate. TEM (Hitachi, HT7700) was then utilized in observing and taking photographs of the exosomes. Equal amounts of plasma exosome proteins were subjected to SDS-PAGE. A human anti-CD63 (Abcam, 1:1000 dilution) antibody was used to detect exosomes.

\section{RNA Isolation, Library Preparation, and circRNA Sequencing}

Total RNA was extracted using TRIzol reagent (Invitrogen Life Technologies) following the manufacturer's procedure. The quality and quantity of RNA were assessed using the NanoDrop ${ }^{\circledR}$ ND-1000 system. RNA integrity should be maintained, without degradation. Total RNA was then treated with a Ribo-Zero rRNA Removal Kit (Illumina, USA) to remove ribosomal RNA (rRNA). RNA libraries were constructed with a TruSeq Stranded Total RNA Library Prep Kit (Illumina, USA) based on the manufacturer's instructions. RNA libraries were controlled for quality and quantity using a Agilent 2100 Analyzer (Agilent, USA). In total, 150 base pair end reads were constructed for library sequencing using an Illumina HiSeq instrument.

\section{CircRNA Sequencing Analysis}

High-throughput whole transcriptome sequencing was then performed, followed by bioinformatic analysis. Low-quality 
reads and $3^{\prime}$ adaptor-trimmings were filtered using the Cutadapt software (v1.9.3). ${ }^{25}$ The remaining high-quality reads were mapped to the genome/transcriptome using the STAR software. ${ }^{26}$ CircRNAs were then identified using DCC software. $^{27}$ circRNAs were annotated using the circBase database (http://www.circbase.org) and Circ2Traits (http:// gyanxet-beta.com/circdb/). edgeR software (v3.16.5) ${ }^{28}$ was then used to normalize data and analyze differences in expression of circRNAs. Significant differential expressions of circRNAs between groups were identified using t-tests and fold changes. $P$-values $\leq 0.05$, with fold changes $\geq 2.0$, were considered statistically significant. The RefSeq database (https://www.biostars.org) was used in finding mRNA coordinates (circRNA-associated genes) based on back-splicing site coordinates of circRNAs as previously reported. ${ }^{13}$ Cluster analysis of different circRNA-associated genes was achieved with GO (http://www.geneontology.org) and KEGG databases (https://www.genome.jp/kegg). The potential target miRNAs of circRNAs were predicted using the TargetScan (http://www.targetscan.org) and miRanda (http:// miranda.org.uk) on the basis of conserved seed-matching sequences.

Target miRNAs were selected for each circRNA in accordance with gene predictions. Target mRNAs for each miRNA were combined with circRNAs for ceRNA analysis. A circRNA-miRNA-mRNA meshwork was then drawn using Cytoscape (3.7.0).

\section{Microarray Data Resource and Preprocessing}

We have searched circRNA expression profiles of LUAD tissues using GEO database and selected GSE101586. Normalized microarray data were then re-analyzed using paired t-tests in order to identify differentially expressed circRNA between LUAD and paired nontumor tissues. $p<$ 0.05 were used as the cutoff criterion.

\section{Cell Culture and Transfection}

The human LUAD cell lines A549, H1975, and H1299 were purchased from ATCC and cultured in RPMI 1640 medium supplemented with $10 \%$ fetal bovine serum (HyClone). Cells were incubated at $37^{\circ} \mathrm{C}$ in an atmosphere containing $5 \% \mathrm{CO}_{2}$. Cells were transfected with a negative control siRNA (si-NC) or si-circ_0102537 (Ribobio, China) in the presence of INTERFERin siRNA/miRNA transfection reagent (Polyplus) according to the manufacturer's instructions.

\section{CCK-8}

The viability of H1299 cells was tested using a Cell Counting Kit-8 (Dojindo, Japan). These cells $\left(1 \times 10^{4}\right)$ were added to the wells of a 96-well plate and cultured for 24, 48, 72, or 96 h. CCK-8 solution was added to each well. After $2 \mathrm{~h}$ incubation, the optical density at $450 \mathrm{~nm}$ was determined using an automatic microplate reader (BioTek, USA).

\section{Apoptosis Assay}

Cells were collected, washed with PBS after centrifugation at $1000 \mathrm{rpm}$ for $5 \mathrm{~min}$, and incubated with $5 \mu \mathrm{L}$ of Annexin V-fluorescein isothiocyanate (FITC) and propidium iodide (BD, Biosciences) to label the apoptotic cells. A flow cytometer (BD FACSAria II, USA) was used to determine the apoptosis rate.

\section{Wound-Healing Assay}

Cells were added to the wells of a 6-well plate and transfected. We introduced scratch wounds with a constant width at the middle of each well using a $200-\mu \mathrm{L}$ pipette tip. We photographed the wells $(0 \mathrm{~h})$ immediately and then after 24-h using a light-microscope (Olympus, Japan).

\section{Transwell Assay}

We placed Transwell assays inserts (Corning, USA) into the wells of a 6-well plate. The membrane in the apical Transwell chamber was coated with Matrigel (BD Biosciences). We added $1 \mathrm{~mL}$ of RPMI 1640 containing $10 \%$ FBS to the bottom chamber and then seeded $4 \times 10^{5}$ cells in $500 \mu \mathrm{L}$ of RPMI 1640 in the upper chamber 24-h after transfection. The cells were fixed in $4 \%$ paraformaldehyde and stained with Giemsa solution 48-h later.

\section{Quantitative Real-Time Polymerase Chain Reaction Analysis}

cDNA synthesis was performed using a PrimeScript RT Reagent Kit (Perfect Real Time; TaKara, Osaka, Japan). Resultant cDNA was used as a template for subsequent realtime PCR amplification. Internal parameters and circRNA candidates of each sample were then subjected to real-time PCR using an Applied Biosystems QuantStudio 3 Real-Time PCR System (Thermo Fisher Scientific), in conjunction with the qPCR SYBR Green master mix (ROX; TaKara). Relative circRNA expression was calculated using the $2^{-\Delta \Delta C T}$ method. Primers for internal parameters of GAPDH, six circRNAs and four mRNAs are provided in Table 2. 


\section{Immunofluorescence Analysis}

Cells were washed twice with PBS, fixed with $4 \%$ paraformaldehyde, and permeabilized with $0.2 \%$ Triton X-100 48-h after transfection. After blocking with 5\% BSA for $30 \mathrm{~min}$, the cells were incubated with an anti-E-cadherin (Proteintech, diluted 1:100) antibody or an anti-vimentin (Proteintech, diluted 1:100) antibody at $4{ }^{\circ} \mathrm{C}$ overnight. The secondary antibody, Alexa Fluor 594-conjugated goat anti-rabbit IgG (Invitrogen, diluted 1:1000), was then added. DAPI (Meilunbio, China) was used to visualize nuclei. The cells were observed using a confocal microscope (Olympus, Japan).

\section{Colony-Formation Assay}

H1299 cells $\left(5 \times 10^{2}\right)$ were transfected with si-NC or sicirc_0102537 and then added to the wells of 6-well plates. After 2 weeks, the colonies were fixed with $4 \%$ paraformaldehyde and stained using Giemsa solution. Then the colonies were counted.

Table 2 Primers and DNA Sequences Used in This Study

\begin{tabular}{|l|l|l|}
\hline CircRNA & $\begin{array}{l}\text { Primer } \\
\text { Type }\end{array}$ & Primer Sequence (5'-3') \\
\hline has_circ_0000972 & $\begin{array}{l}\text { Forward } \\
\text { Reverse }\end{array}$ & $\begin{array}{l}\text { TCTTTGACTATGGACAATATTCTGC } \\
\text { TGAATGTAGATAAGTTCGAAACCA }\end{array}$ \\
\hline has_circ_0001769 & $\begin{array}{l}\text { Forward } \\
\text { Reverse }\end{array}$ & $\begin{array}{l}\text { TGCCAGATGGAAGAACCATT } \\
\text { CAGTAACAAAAAGCGCAGAGC }\end{array}$ \\
\hline has_circ_0001578 & $\begin{array}{l}\text { Forward } \\
\text { Reverse }\end{array}$ & $\begin{array}{l}\text { TAGCATCAGGCAAAGGAAGC } \\
\text { CATCGACCACTTCTCCTGGT }\end{array}$ \\
\hline has_circ_0001070 & $\begin{array}{l}\text { Forward } \\
\text { Reverse }\end{array}$ & $\begin{array}{l}\text { TCATTCCACCTGGTCAACAA } \\
\text { TTGGCCACTGACTAGGCTCT }\end{array}$ \\
\hline has_circ_0000647 & $\begin{array}{l}\text { Forward } \\
\text { Reverse }\end{array}$ & $\begin{array}{l}\text { CCGAGGCCTCTATAAACAAGG } \\
\text { GCCGATGAGACAATCATTCC }\end{array}$ \\
\hline has_circ_0102537 & $\begin{array}{l}\text { Forward } \\
\text { Reverse }\end{array}$ & $\begin{array}{l}\text { GCCCATGCCAAATAGTTACC } \\
\text { TGCTTTTGAGTGCAACTTCAG }\end{array}$ \\
\hline E-cadherin & $\begin{array}{l}\text { Forward } \\
\text { Reverse }\end{array}$ & $\begin{array}{l}\text { CGGGAATGCAGTTGAGGATC } \\
\text { AGGATGGTGTAAGCGATGGC }\end{array}$ \\
\hline N-cadherin & $\begin{array}{l}\text { Forward } \\
\text { Reverse }\end{array}$ & $\begin{array}{l}\text { AGCCTGGAACATATGTGATGA } \\
\text { CCATAAAACGTCATGGCAGTAA }\end{array}$ \\
\hline Snail & $\begin{array}{l}\text { Reverwerse } \\
\text { Reverse }\end{array}$ & $\begin{array}{l}\text { GAAAGGCCTTCAACTGCAAA } \\
\text { TGACATCTGAGTGGGTCTGG } \\
\text { TCCTCCGCCTCCTGCAGGTTCTT }\end{array}$ \\
\hline AGGGGAGATTCAGTGTGGTG
\end{tabular}

\section{Statistical Analysis}

Data are expressed as mean \pm SD. Between-group differences were analyzed using Student's $t$-test. All statistical analyses were performed using SPSS software, ver. 21.0. A two-sided $p$-value $<0.05$ was considered statistically significant.

\section{Results Detection of Exosomes}

TEM and Western blot analysis were used in identifying the exosomes. Under TEM, exosomes were observed as irregular spheres between 30 and $100 \mathrm{~nm}$ in diameter, with a clear and intact membrane (Supplemental Fig. S1A). Western blotting demonstrated that the exosomes were enriched with typical exosomal marker, CD63 (Supplemental Fig. S1B). These analyses indicate that the plasma-derived particles isolated from study subjects are exosomes.

\section{Differentially Expressed circRNAs}

Plasma circRNAs from five advanced-stage LUAD patients and five healthy subjects were sequenced. In total, 21,917 exosomal circRNAs were identified. These circRNAs were further sorted into five categories based on relationships with coding genes: $58.15 \%$ $(12,745 / 21,917)$ were exonic circRNAs, $18.88 \%$ (4137/ $21,917)$ intronic circRNAs, $11.11 \%(2436 / 21,917)$ were sense-overlapping circRNAs, $8.4 \%(1842 / 21,917)$ were antisense circRNAs, and 3.45\% $(757 / 21,917)$ intergenic circRNAs (Supplemental Fig. S2A). In total, 10,051 circRNAs were identified as novel circRNAs, whereas the other 11,866 circRNAs are circBase circRNAs ( $\mathrm{n}=$ $11,685)$ and literature circRNAs $(\mathrm{n}=181)$ (Supplemental Fig. S2B). The distribution of circRNA in human chromosomes has been illustrated in Supplemental Fig. S2C. Most are located on Chr 1,

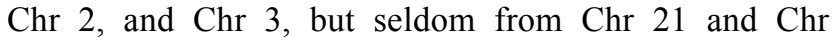
22. A hierarchical clustering map, volcano plot, and scatter plot have shown that the circRNA expression levels are distinguished and clustered in patients with advanced-stage LUAD and control participants, respectively (Figure 1A-C). Thus, 220 circRNAs were differentially regulated by fold change $\geq 2.0$ and $p<0.05$. In total, 214 circRNAs were downregulated and 6 were upregulated. Ten circRNAs are reported for the first time; 210 are previously identified molecules. Meanwhile, 207 were identified to be exonic circRNAs, 6 were intronic circRNAs, 5 were sense- 
A

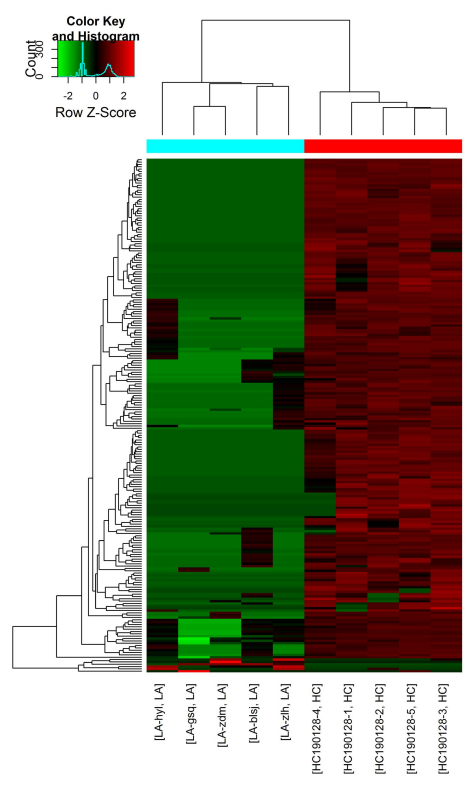

B

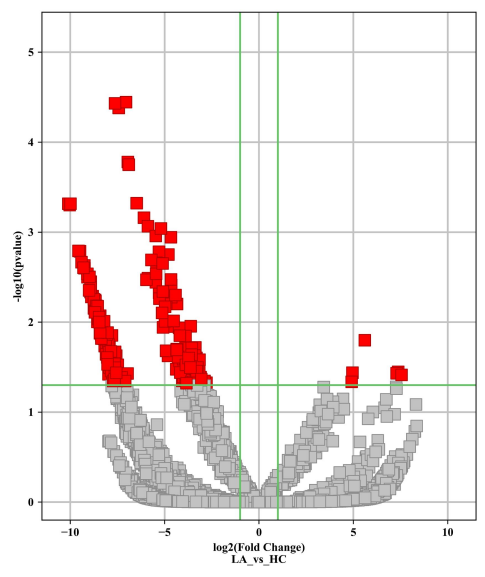

C

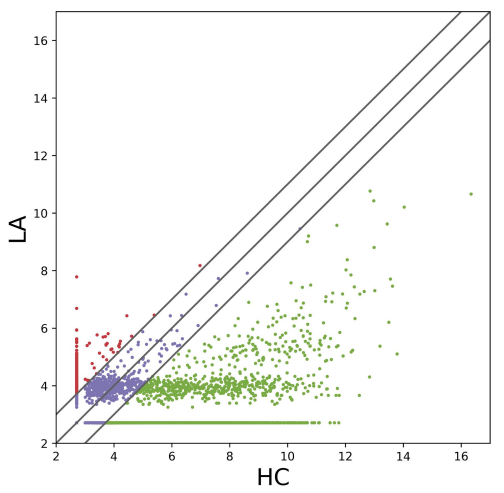

Figure I Differential expression of circRNAs in plasma exosomes from patients with LUAD. (A) Heat map with each column representing a blood sample and each row representing a differentially expressed circRNA. Red color represents the upregulated circRNAs, whereas green represents downregulated circRNAs. (B) Volcano plots of differential expression of circRNAs between LUAD and normal control plasma samples. The red squares show the differentially expressed circRNAs identified by circRNA sequencing. The vertical line marks the twofold (log2 scaled) up or down changes. The horizontal line marks the $p$-value of 0.05 ( $-\log 10$ scaled). (C) Scatter plots of the distribution of circRNAs.

overlapping circRNAs, and 2 were antisense circRNAs. Table 3 lists six upregulated circRNAs and the top 10 downregulated circRNAs. All of these circRNAs met the above criteria for significance.

\section{GO and KEGG Pathway Analyses}

To better understand the functions of the differentially expressed circRNAs, we mapped all of them using GO and KEGG enrichment analyses. GO analysis has been identified to consist three ontologies, namely, molecular functions, cellular components, and biological processes (Figure 2). GO analysis indicated that BP of differentially expressed circRNAs was mainly involved in the regulation of centrosome duplication, centrosome cycle, and protein expert from the nucleus in the upregulated circRNA group. In downregulated groups, differentially expressed circRNAs were found to be primarily related to small GTPase-mediated signal transduction, GTP catabolic process, and guanosine-containing compound catabolic process. GO analysis of molecular functions suggested that the upregulated differentially expressed circRNAs were correlated with nucleocytoplasmic transporter activity, Ran GTPase binding, and protein transporter activity. Binding, nucleotide-binding, and nucleoside phosphatebinding have been determined to share a significant amount of differentially expressed circRNAs with "molecular function" GO term in downregulated groups. Within cellular components, these upregulated circRNAs were mainly enriched in the autophagic vacuole membrane, preautophagosomal structure, and endoplasmic reticulum Golgi intermediate compartment membrane. In downregulated groups, intracellular part, intracellular, and cytosol were primary enriched categories. Furthermore, KEGG enrichment analysis has revealed the roles of the predicted circRNA-target mRNAs and further predicted that the changes in circRNAs affected the signaling pathways. As illustrated in Figure 3, the upregulated circRNAs were mainly concentrated in RNA transport, ribosome biogenesis in eukaryotes, and influenza A. Intriguingly, VEGF signaling pathway, vascular smooth muscle contraction, and RAS signaling pathway were determined to be the three significantly enriched terms in KEGG pathways among the downregulated circRNAs, indicating that these predicted target genes were related to tumor signaling pathways.

\section{Identification of the circRNA-miRNA- mRNA Network}

One important function of circRNAs is to sponge miRNA, which has been determined to subsequently modulate the 
Table 3 The 6 Upregulated and the Top 10 Downregulated Exosomal circRNAs

\begin{tabular}{|c|c|c|c|c|c|}
\hline CircRNA ID & Log FC & $\mathbf{P}$ & CircBase ID & Gene Name & Catalog \\
\hline chr6:86,235,844-86,25I,76I- & $7.56|05947|$ & 0.038637734 & hsa_circ_0001616 & SNXI4 & exonic \\
\hline chrl7:57,808,782-57,85I,246+ & 7.377508588 & 0.035715912 & hsa circ 0005077 & VMPI & exonic \\
\hline chrY:I3,464,932-13,479,648- & 7.297925452 & 0.037091459 & & G090815 & intronic \\
\hline $\operatorname{chrl}: 22,8 \mid 6,373-22,818,026+$ & 5.611143385 & 0.015931314 & hsa_circ_0007418 & ZBTB40 & exonic \\
\hline chr6:86,227,474-86,239,993- & 4.954648405 & 0.036548837 & hsa_circ_0008093 & SNXI4 & exonic \\
\hline chr2:6I,722,590-6I,726,048- & 4.913980046 & 0.046170865 & hsa_circ_0054886 & XPOI & exonic \\
\hline chr3:50, I45,503-50, I45,737+ & -10.095272994 & 0.000484406 & hsa_circ_000I305 & RBM5 & exonic \\
\hline chrl:9,991,949-9,994,918- & -10.025287824 & 0.000501802 & hsa_circ_0000014 & LZIC & exonic \\
\hline chr I7:26,490,569-26,499,644+ & $-9.98 \mid 447398$ & 0.000484323 & hsa_circ_0003638 & $N L K$ & exonic \\
\hline chrl:33,760,538-33,760,906+ & -9.556098469 & 0.001619763 & hsa_circ_0009027 & ZNF362 & exonic \\
\hline chrl9:57,967,02I-57,967,550- & $-9.47864894 I I$ & 0.001634728 & hsa_circ_0000963 & AC004076.9 & exonic \\
\hline chr3:|43,5|3,843-|43,5|5,745- & -9.4004065635 & 0.002159249 & hsa_circ_0067678 & SLC9A9 & exonic \\
\hline chr7:134,642,820-134,643,04|+ & -9.2955750926 & 0.002489644 & & CALDI & exonic \\
\hline chr3:196, II 8,684-196, I20,490- & -9.2487518653 & $0.002348181 I$ & hsa_circ_000505 & UBXN7 & exonic \\
\hline $\operatorname{chrl6:24,043,457-24,046,868+}$ & -9.24294194001 & 0.0025234182 & hsa_circ_0000682 & $P R K C B$ & exonic \\
\hline chrl7:35,800,606-35,800,763+ & -9.09187322767 & 0.0029134626 & hsa circ 0006220 & TADA2A & exonic \\
\hline
\end{tabular}

expression of target mRNA. Hence, we selected 14 downregulated circRNAs in predicting possible interactions between circRNAs, miRNAs, and mRNA based on fold change, $p$-value, and known function of the circRNAs. The obtained circRNA-miRNA-mRNA network (Supplemental Fig. S3) is complicated and further shows that miR-7161-3p and miR-103a-2-5p are regulated by more circRNAs than other miRNAs.

\section{CircRNA Validation by qRT-PCR}

To confirm the authenticity of the globally downregulated circRNAs, we randomly selected five differentially expressed circRNAs (has_circ_0000972, hsa_circ_0001769, hsa_circ_0001578, hsa_circ_0001070, and hsa_circ_0000647), which were subjected to qRT-PCR in five pairs of sequenced LUAD and healthy samples. All circRNAs were significantly downregulated in plasma exosomes of LUAD patients

A
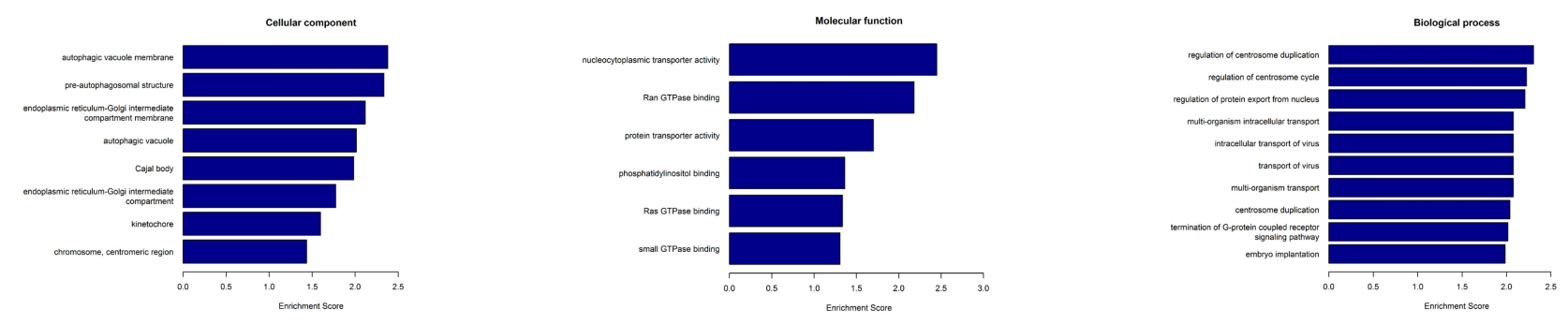

B
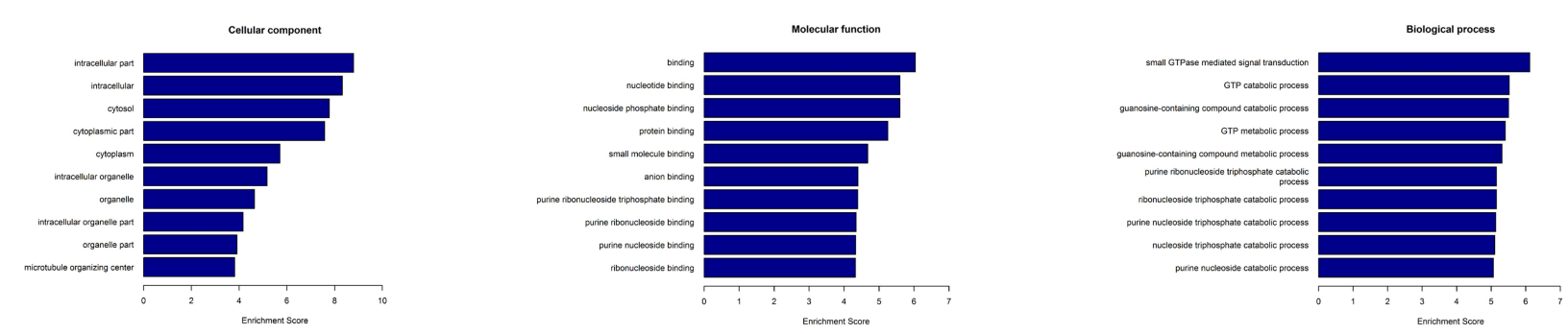

Figure 2 Gene ontology enrichment analysis. The horizontal axis represents the enrichment score. The vertical axis represents GO terms. (A) GO analysis of the upregulated circRNAs. (B) GO analysis of the downregulated circRNAs. 
A

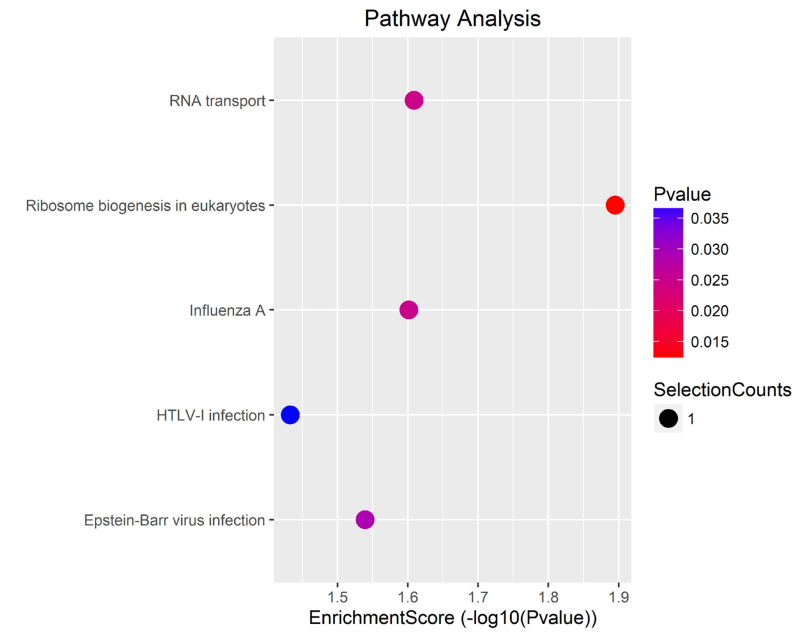

B

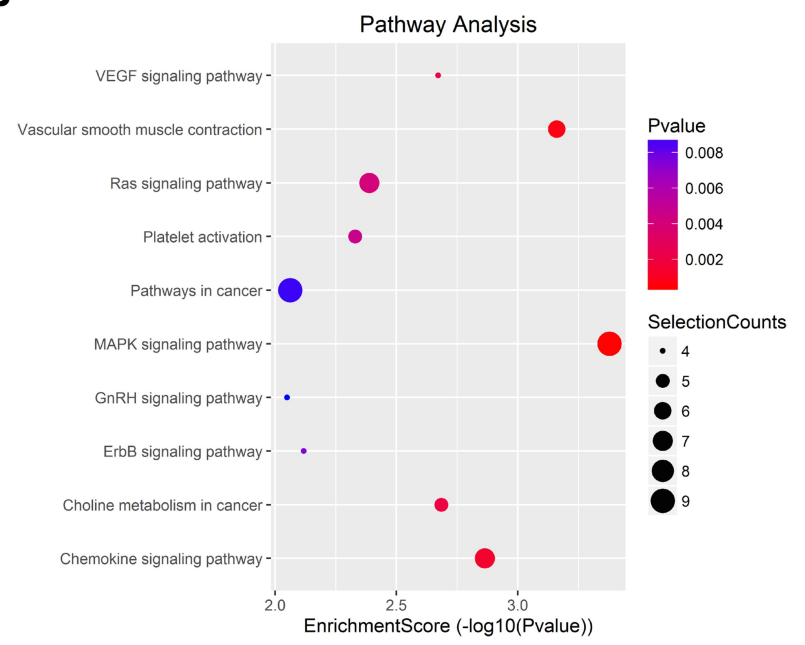

Figure 3 KEGG pathway analysis. The spot size represents the number of genes associated with the listed pathway, while the color refers to the $p$-value. (A) KEGG pathways analysis of significantly upregulated circRNAs. (B) KEGG pathway analysis of significantly downregulated circRNAs.

compared with those of healthy controls, confirming the reliability of the circRNA-seq data (Figure 4).

\section{Identification of Differentially Expressed circRNAs Both in LUAD Plasma Exosome and Tissues}

Microarray datasets GSE101516 were included in this study to identify differentially expressed circRNAs in LUAD tissues. We examined the intersections of differentially expressed circRNAs and GSE101516. The circRNA, hsa_circ 0102537 , was found to be downregulated in both LUAD plasma exosomes and tissues (Figure 5A). A ceRNA network was constructed to predict its function (Figure 5B), and GO and KEGG analyses (Figure 5C) were

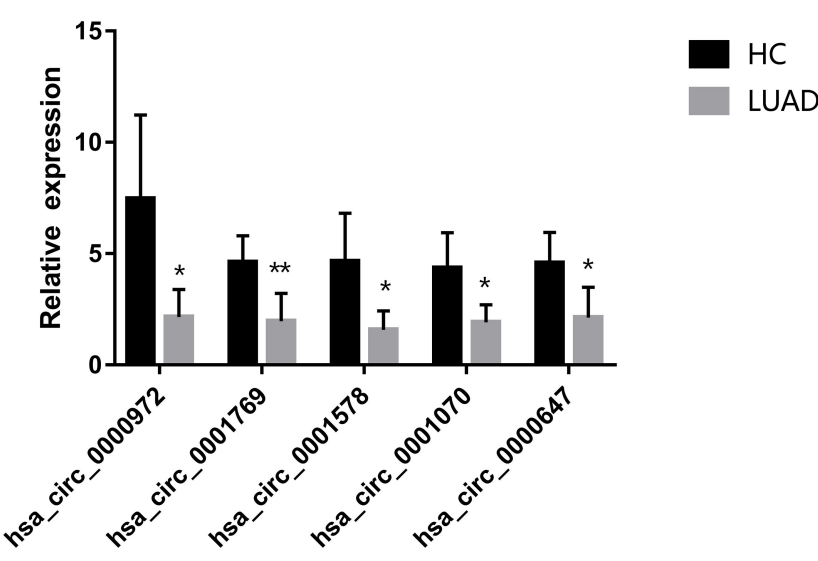

Figure 4 Validation of the differentially expressed circRNAs by qRT-PCR. Five downregulated circRNAs were selected. Bars represent the mean $\pm \operatorname{SEM}(n=5)$. $*_{p}<0.05, * *_{p}<0.01$. then performed. Based on the CircInteractome prediction, targeted miRNA of hsa_circ_0102537 were identified. We then predicted the mRNA targeted by these miRNAs using the TargetScan. The credibility of the network was improved by only retaining circRNA-miRNA-mRNA gene sets in which circRNA and mRNA are positively correlated. GO analysis of hsa_circ_0102537 was mainly enriched in intracellular and organelle processes, especially membranebounded organelles. KEGG analysis retrieved two cancerrelated pathways, namely, fluid shear stress and PI3K-Akt signaling pathway. Both of these pathways are deemed to be worthy of special attention as they provide more credibility on the assumption that hsa_circ_0102537 may play a crucial role in LUAD initiation and progression. Considering the important value of hsa_circ_0102537, its expression was validated in all 13 LUAD and 12 healthy plasma samples. The qRT-PCR results have shown the significant downregulation of hsa_circ_0102537 in LUAD plasma exosomes (Figure 5D).

\section{Circ_0102537 Inhibits the Migration and Invasion of LUAD Cells}

The expression of circ_0102537 in LUAD cell lines in vitro was measure using qRT-PCR. Circ_0102537 was upregulated the most in H1299 cells (Figure 6A). We next designed circ_0102537-specific siRNAs (Figure 6B). Circ_0102537\#1 was the most effective siRNA and was selected for subsequent experiments.

The roles of circ_0102537 in the progression of LUAD were determined using wound healing and Transwell 
A

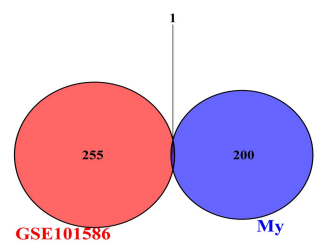

B

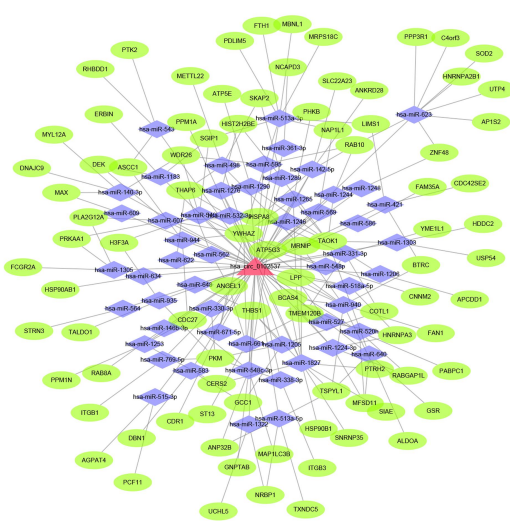

C

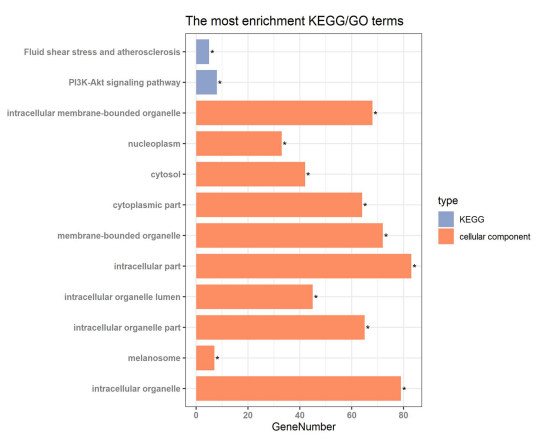

D

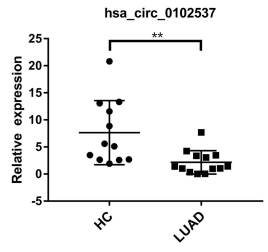

Figure 5 Identification of differentially expressed circRNAs in LUAD plasma exosome and tissues. (A) Identification of overlapping differentially expressed circRNA in RNAseq and GSEI01586. (B) GO and KEGG analysis of hsa_circ_0102537. (C) Construction of circRNA-miRNA-mRNA meshwork of hsa_circ_0102537. (D) Expression of hsa_circ_102537 in plasma exosomes. $*_{p}<0.05, * *_{p}<0.01$.

assays. We found that inhibiting the expression of circ_0102537 promoted cell migration and invasion (Figure 6C and D). Immunofluorescence and qRT-PCR assays were used to detect markers of the epithelialmesenchymal transition expressed by H1299 cells in which circ_0102537 expression was inhibited. These analyses revealed that E-cadherin expression was decreased, and vimentin was increased in these cells. Further, qRTPCR assays demonstrated that the expression of $\mathrm{N}$-cadherin and snail had increased (Figure 6E and F). However, circ_0102537 did not affect cell proliferation, apoptosis, and colony formation (Supplemental Fig. S4).

\section{Discussion}

Lung adenocarcinoma has been identified as the most frequent subtype of lung cancer, and its mortality in advanced stages has been recorded to be high. Even with regular radiographic screening, most cases of LUAD are diagnosed at advanced stages. Thus, novel biomarkers are needed in optimizing future treatment for this malignancy. Previous studies report that circRNAs play an important role in the occurrence and development of tumors. Tumor cells constantly reportedly release exosomes during tumor development and progression. These exosomes regulate cell proliferation, angiogenesis, thrombosis, invasion, and immunosuppression. ${ }^{29}$ CircRNAs are stably present in exosomes; thus, they could serve as novel biomarkers that can be measured non-invasively. The circRNAs present in the exosomes could act as messengers in cell-tocell communication to regulate functions in the target cells. ${ }^{30}$ However, studies focusing on extending the pattern and potential role of circRNAs in plasma exosome in LUAD patients remain to be limited. The manuscript of Chen et al is the only publication that provides an initial landscape for differential expression of exosomal circRNA in plasma from early-stage lung adenocarcinoma. ${ }^{31}$ However, most patients with LUAD are diagnosed at advanced or metastatic stages, when treatment options are limited and prognosis is poor. In our study, the expression profiles of exosomal circRNAs between advanced-stage LUAD patients and healthy individuals were analyzed for the first time using high-throughput whole transcriptome sequencing. Identifying differentially expressed exosomal circRNAs has been determined to shed some light on intermolecular interactions and signaling pathways involved in advanced-stage LUAD. Western blot analysis and TEM were also used to confirm exosomes isolated from the peripheral blood. In this study, we found 220 putative circRNA candidates derived from 21,917 genes.

Several studies have provided evidence that differentially expressed circRNAs in tumor tissues or plasma exosomes of NSCLC patients are involved in cancer-related signaling. ${ }^{31-33}$ In total, 214 downregulated circRNAs were identified, with only 6 upregulated circRNAs. Our result was not consistent with exosomal circRNA differential expression in early-stage lung adenocarcinoma that indicates a relatively higher number of upregulated circRNAs than downregulated circRNAs. ${ }^{31}$ Thus, circRNAs share not only common expression patterns but also exhibit distinct circRNA expression signatures between early and advanced-stage LUAD.

Due to their circular structure, circRNAs are resistant to RNase R and RNA exonuclease degradation. Further, compared in cells, concentrations of circRNA in exosomes were 
A

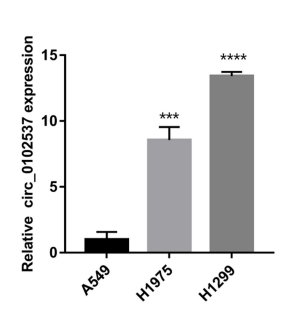

D

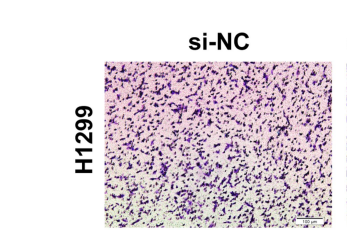

B

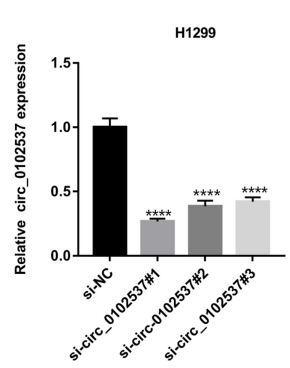

C
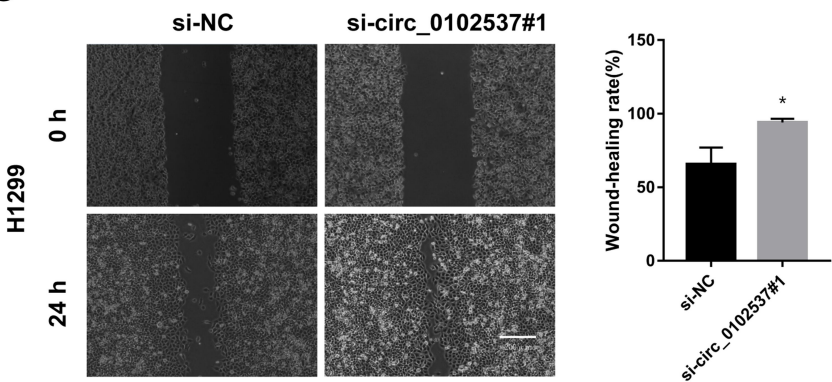

E

F
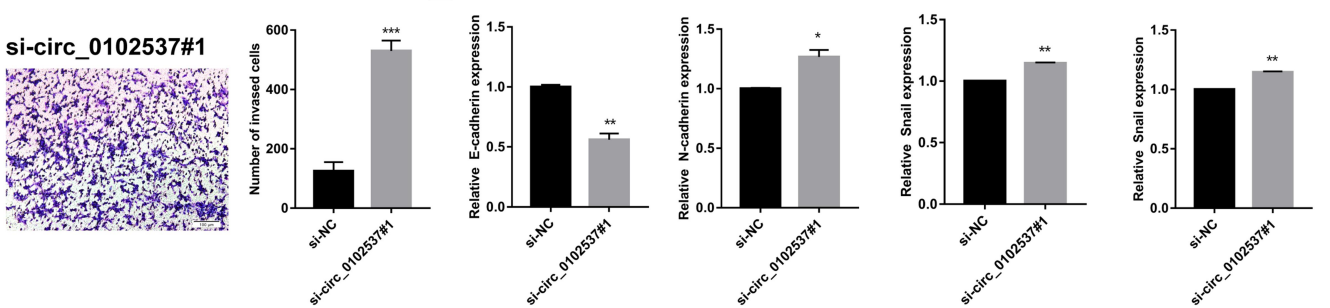

DAPI

E-cadherin

DAPI
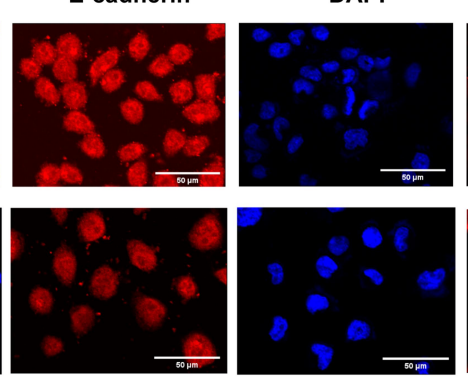

Vimentin
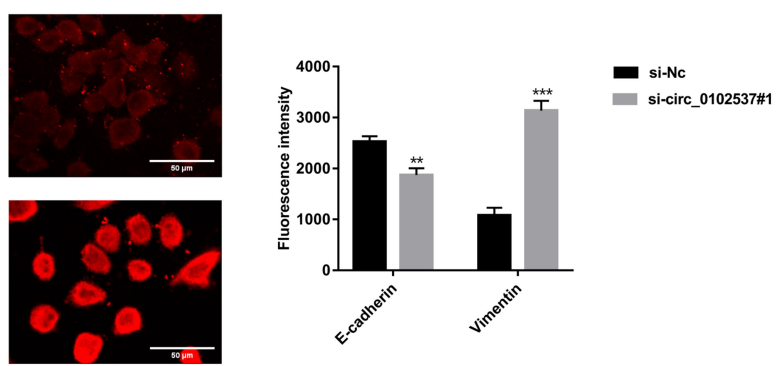

Figure 6 Circ_0102537 inhibits the migration and invasion of HI299 cells in vitro. (A) QRT-PCR was performed to verify the expression of circ_0102537 in the human LUAD cell lines. (B) HI299 cells were transfected with si-NC, si-circ_0102537\#I, si-circ_0102537\#2 or si-circ_0102537\#3. qRT-PCR assays confirmed that the transfection was successful. Wound healing assay (C) to assess cell migration. Transwell assay (D) measured invasion of Matrigel by HI299 cells transfected with si-NC or si-circ _0102537\#I. HI299 transfected with si-NC or si-circ_0102537\#I were subjected to QRT-PCR (E) and immunofluorescence (F) assays to measure the expression levels of EMT markers. $* p<0.05, * * p<0.01$, *** $p<0.001$, ***** $p 0.000$ I.

determined to be greater. ${ }^{34}$ Exosomal circRNAs may prove to be diagnostic biomarkers for lung cancer, because of their abundance, stability, and specificity. ${ }^{35}$ For example, plasma circ_0013958 distinguished LUAD patients from healthy controls with an area under the receiver operating characteristic (AUC) of $0.794(95 \% \mathrm{CI}=0.703-0.912 ; \mathrm{P}<0.001)$. circ_0013958 in plasma could be an accessible noninvasive biomarker for LUAD. ${ }^{36}$ Further, recent research has demonstrated that exosomal circRNAs affect LUAD development and progression by modulating the activation of LUAD-associated molecules and pathways and may be used as LUAD markers for treatment. ${ }^{22-24}$

GO and KEGG pathway analyses were used to further understand the potential role of the differentially expressed circRNAs. These genes were assessed using GO function analysis to annotate likely functions. This analysis indicated an association with binding of specific molecules, such as nucleotides, GTPase, proteins, and small molecules.
Additionally, circRNAs were also found to be correlated with nucleocytoplasmic/protein transporter activity. For cellular components, significant correlations were found with autophagic vacuole and intracellular structures, such as autophagosome, cytosol, and organelle. Key biological processes include regulation of centrosome duplication and cycle and GTP metabolism. These results are consistent with previous studies, demonstrating that autophagy, ${ }^{37}$ centrosome abnormalities, ${ }^{38}$ and DNA damage ${ }^{39}$ are involved in the pathophysiology of LUAD.

KEGG pathway analysis indicated that differentially expressed circRNAs correspond to tumor-related signaling pathways. The strongest correlation was determined to be with vascular endothelial growth factor (VEGF) signaling. Angiogenesis is recognized as an important facilitator of tumor growth, invasion, and metastases. VEGF is an important mediator for stimulating tumor angiogenesis. Novel drugs that target VEGF, including anti-VEGF antibodies, anti-VEGF 
receptor antibodies, and small-molecule tyrosine kinase inhibitors, are already available and approved for the treatment of various tumors, including lung cancer. ${ }^{40}$ Besides, a large number of downregulated differentially expressed circRNAs have been associated with other tumor signaling pathways, such as platelet activation, ${ }^{41}$ MAPK signaling, ${ }^{42}$ and RAS signaling. ${ }^{43}$ KEGG analysis showed that upregulated and differentially expressed circRNAs were mainly associated with RNA transport and ribosome biogenesis with potential targets for cancer treatment. ${ }^{44,45}$ In recent years, carcinogenic effect of viruses has become a hot topic in the etiology of lung cancer. ${ }^{46}$ These findings are found to be consistent with our results that influenza A, HTLV-I, and Epstein-Barr virus infection may be correlated with the development of lung cancer. We hypothesized that differentially expressed circRNAs may modulate activation of the tumor signaling pathways, which in turn affect LUAD development and progression. Thus, circRNAs in plasma exosomes might be used as LUAD markers for diagnosis and treatment.

Recent functional studies have suggest that circRNAs may function as miRNA sponges. ${ }^{47}$ miRNAs are highly conserved, which negatively regulated target mRNAs. The circRNA-miRNA-mRNA network was built based on our RNA-seq data that predict how circRNAs affect LUAD occurrence and progression. Some miRNA targets associated with the circRNAs were reported for LUAD. For example, miR-181a-5p inhibits A549 cell proliferation and invasion by targeting Kras. ${ }^{48}$ MiR-544a promotes lung cancer cell migration by targeting cadherin $1 .{ }^{49}$ Further, mir-450b-5p, ${ }^{50}$ mir$330-3 p,{ }^{51}$ and mir-335-3p $p^{52}$ are determined to be prominent in tumorigenesis and progression.

The use of microarray GSE101516 for four paired LUAD and nontumor lung tissues further clarified the function of circRNAs in the development of LUAD. A combination of our RNA-seq and microarray analysis revealed that has_circ_0102537 is significantly downregulated in both datasets. We have also analyzed hsa_circ_0102537 involvement in two cancer-related signaling pathways, fluid shear stress, ${ }^{53}$ and PI3K-Akt signaling pathway. ${ }^{54}$ The PI3K/AKT pathway is implicated in lung cancer initiation and progression. For NSCLC, a few specific inhibitors of PI3K and Akt are currently in various phases of preclinical investigation and even in early phase clinical trials. ${ }^{55}$ Further experiments showed that circ_0102537 functioned as a tumor suppressor by inhibiting the migration and invasion of LUAD cells in vitro, albeit through an unknown mechanism. Therefore, we will focus on the interaction between circ_0102537miRNA and its mRNA targets in the future. Circ_0102537 may be a promising diagnostic biomarker. Circ_0102537 may be a therapeutic target for the metastasis of LUAD.

\section{Conclusion}

In summary, this present study extends current knowledge as regards differentially expressed circRNAs in plasma exosomes of advanced-stage LUAD. Our results suggest promising avenues for further research into LUAD development and biomarkers for diagnosis and prognosis. Circ_0102537 may prove useful as a diagnostic biomarker for advanced-stage LUAD.

\section{Abbreviations}

EMT, Epithelial-mesenchymal transition; GEO, Gene Expression Omnibus; GO, Gene ontology; KEGG, Kyoto Encyclopedia of Genes and Genomes; LUAD, Lung adenocarcinoma; LUSC, Lung squamous cell carcinoma; NSCLC, Non-small cell lung cancer; SCLC, Small cell lung cancer; TEM, Transmission electron microscopy; VEGF, Vascular endothelial growth factor.

\section{Disclosure}

The authors report no conflicts of interest for this work.

\section{References}

1. Bray F, Ferlay J, Soerjomataram I, Siegel RL, Torre LA, Jemal A. Global cancer statistics 2018: GLOBOCAN estimates of incidence and mortality worldwide for 36 cancers in 185 countries. CA Cancer J Clin. 2018;68(6):394-424. doi:10.3322/caac.21492

2. Mao Y, Yang D, He J, Krasna MJ. Epidemiology of Lung Cancer. Surg Oncol Clin N Am. 2016;25(3):439-445. doi:10.1016/j.soc.2016.02.001

3. Zappa C, Mousa SA. Non-small cell lung cancer: current treatment and future advances. Translational lung cancer research. 2016;5 (3):288-300. doi:10.21037/tlcr.2016.06.07

4. Wang M, Yu F, Li P, Wang K. Emerging Function and Clinical Significance of Exosomal circRNAs in Cancer. Mol Ther Nucleic Acids. 2020;21:367-383. doi:10.1016/j.omtn.2020.06.008

5. Pegtel DM, Gould SJ. Exosomes. Annu Rev Biochem. 2019;88:487-514.

6. Thery C, Witwer KW, Aikawa E, et al. Minimal information for studies of extracellular vesicles 2018 (MISEV2018): a position statement of the International Society for Extracellular Vesicles and update of the MISEV2014 guidelines. J Extracellular Vesicles. 2018;7(1):1535750.

7. Li S, Li Y, Chen B, et al. exoRBase: a database of circRNA, IncRNA and mRNA in human blood exosomes. Nucleic Acids Res. 2018;46 (D1):D106-D112. doi:10.1093/nar/gkx891

8. Wei Z, Batagov AO, Schinelli S, et al. Coding and noncoding landscape of extracellular RNA released by human glioma stem cells. Nat Commun. 2017;8(1):1145.

9. Zhou L, Lv T, Zhang Q, et al. The biology, function and clinical implications of exosomes in lung cancer. Cancer Lett. 2017;407:84-92. doi:10.1016/j.canlet.2017.08.003

10. Wang Y, Yi J, Chen X, Zhang Y, Xu M, Yang Z. The regulation of cancer cell migration by lung cancer cell-derived exosomes through TGF-beta and IL-10. Oncol Lett. 2016;11(2):1527-1530. doi:10.3892/ol.2015.4044 
11. You J, Li M, Cao LM, et al. Snaill-dependent cancer-associated fibroblasts induce epithelial-mesenchymal transition in lung cancer cells via exosomes. QJM. 2019;112(8):581-590. doi:10.1093/qjmed/ hcz093

12. Lei Y, Guo W, Chen B, Chen L, Gong J, Li W. Tumorreleased lncRNA H19 promotes gefitinib resistance via packaging into exosomes in nonsmall cell lung cancer. Oncol Rep. 2018;40 (6):3438-3446.

13. Memczak S, Jens M, Elefsinioti A, et al. Circular RNAs are a large class of animal RNAs with regulatory potency. Nature. 2013;495 (7441):333-338. doi:10.1038/nature11928

14. Li Z, Huang C, Bao C, et al. Exon-intron circular RNAs regulate transcription in the nucleus. Nat Struct Mol Biol. 2015;22 (3):256-264. doi:10.1038/nsmb.2959

15. Broadbent KM, Broadbent JC, Ribacke U, Wirth D, Rinn JL, Sabeti PC. Strand-specific RNA sequencing in Plasmodium falciparum malaria identifies developmentally regulated long non-coding RNA and circular RNA. BMC Genomics. 2015;16:454. doi:10.1186/ s12864-015-1603-4

16. Salzman J, Chen RE, Olsen MN, Wang PL, Brown PO. Cell-type specific features of circular RNA expression. PLoS Genet. 2013;9(9): e1003777. doi:10.1371/journal.pgen.1003777

17. Chen LL. The expanding regulatory mechanisms and cellular functions of circular RNAs. Nat Rev Mol Cell Biol. 2020;21:475-490. doi:10.1038/s41580-020-0243-y

18. Huang X, Wu B, Chen M, et al. Depletion of exosomal circLDLR in follicle fluid derepresses miR-1294 function and inhibits estradiol production via CYP19A1 in polycystic ovary syndrome. Aging. 2020;10:12.

19. Han J, Zhang L, Hu L, et al. Circular RNA-Expression Profiling Reveals a Potential Role of Hsa_circ_0097435 in Heart Failure via Sponging Multiple MicroRNĀs. Front Genet. 2020;11:212. doi:10.3389/fgene. 2020.00212

20. Zhao RT, Zhou J, Dong XL, et al. Circular Ribonucleic Acid Expression Alteration in Exosomes from the Brain Extracellular Space after Traumatic Brain Injury in Mice. $J$ Neurotrauma. 2018;35(17):2056-2066. doi:10.1089/neu.2017.5502

21. Lei M, Zheng G, Ning Q, Zheng J, Dong D. Translation and functional roles of circular RNAs in human cancer. Mol Cancer. 2020;19 (1):30. doi:10.1186/s12943-020-1135-7

22. Ma J, Qi G, Li L. A Novel Serum Exosomes-Based Biomarker hsa_circ_0002130 Facilitates Osimertinib-Resistance in Non-Small Cell Lung Cancer by Sponging miR-498. Onco Targets Ther. 2020;13:5293-5307. doi:10.2147/OTT.S243214

23. Wang J, Zhao X, Wang Y, et al. circRNA-002178 act as a ceRNA to promote PDL1/PD1 expression in lung adenocarcinoma. Cell Death Dis. 2020;11(1):32.

24. Zhang N, Nan A, Chen L, et al. Circular RNA circSATB2 promotes progression of non-small cell lung cancer cells. Mol Cancer. 2020;19 (1):101. doi:10.1186/s12943-020-01221-6

25. Kechin A, Boyarskikh U, Kel A, Filipenko M. cutPrimers: A New Tool for Accurate Cutting of Primers from Reads of Targeted Next Generation Sequencing. J computational biol. 2017;24 (11):1138-1143. doi:10.1089/cmb.2017.0096

26. Dobin A, Davis CA, Schlesinger F, et al. STAR: ultrafast universal RNA-seq aligner. Bioinformatics. 2013;29(1):15-21. doi:10.1093/ bioinformatics/bts635

27. Cheng J, Metge F, Dieterich C. Specific identification and quantification of circular RNAs from sequencing data. Bioinformatics. 2016;32 (7):1094-1096. doi:10.1093/bioinformatics/btv656

28. Robinson MD, McCarthy DJ, Smyth GK. edgeR: a Bioconductor package for differential expression analysis of digital gene expression data. Bioinformatics. 2010;26(1):139-140. doi:10.1093/bioinformatics/btp616

29. Whiteside TL. Tumor-Derived Exosomes and Their Role in Cancer Progression. Adv Clin Chem. 2016;74:103-141.
30. Wang Y, Liu J, Ma J, et al. Exosomal circRNAs: biogenesis, effect and application in human diseases. Mol Cancer. 2019;18(1):116. doi:10.1186/s12943-019-1041-z

31. Chen F, Huang C, Wu Q, Jiang L, Chen S, Chen L. Circular RNAs expression profiles in plasma exosomes from early-stage lung adenocarcinoma and the potential biomarkers. J Cell Biochem. 2020;121 (3):2525-2533. doi:10.1002/jcb.29475

32. Zhang S, Zeng X, Ding T, et al. Microarray profile of circular RNAs identifies hsa_circ_0014130 as a new circular RNA biomarker in non-small cell lung cancer. Sci Rep. 2018;8(1):2878. doi:10.1038/ s41598-018-21300-5

33. Zhao J, Li L, Wang Q, Han H, Zhan Q, Xu M. CircRNA Expression Profile in Early-Stage Lung Adenocarcinoma Patients. Cell Physiol Biochem. 2017;44(6):2138-2146. doi:10.1159/000485953

34. Li Y, Zheng Q, Bao C, et al. Circular RNA is enriched and stable in exosomes: a promising biomarker for cancer diagnosis. Cell Res. 2015;25(8):981-984. doi:10.1038/cr.2015.82

35. Shi X, Wang B, Feng X, Xu Y, Lu K, Sun M. circRNAs and Exosomes: A Mysterious Frontier for Human Cancer. Molecular Therapy Nucleic Acids. 2019;19:384-392. doi:10.1016/j.omtn.2019. 11.023

36. Zhu X, Wang X, Wei S, et al. hsa_circ_0013958: a circular RNA and potential novel biomarker for lung adenocarcinoma. FEBS J. 2017;284(14):2170-2182. doi:10.1111/febs.14132

37. Liu G, Pei F, Yang F, et al. Role of Autophagy and Apoptosis in Non-Small-Cell Lung Cancer. Int J Mol Sci. 2017;18:2.

38. Koutsami MK, Tsantoulis PK, Kouloukoussa M, et al. Centrosome abnormalities are frequently observed in non-small-cell lung cancer and are associated with aneuploidy and cyclin E overexpression. J Pathol. 2006;209(4):512-521. doi:10.1002/path.2005

39. Barnes JL, Zubair M, John K, Poirier MC, Martin FL. Carcinogens and DNA damage. Biochem Soc Trans. 2018;46(5):1213-1224. doi:10.1042/BST20180519

40. Malapelle U, Rossi A. Emerging angiogenesis inhibitors for non-small cell lung cancer. Expert Opin Emerg Drugs. 2019;24 (2):71-81. doi:10.1080/14728214.2019.1619696

41. Hoang LT, Domingo-Sabugo C, Starren ES, et al. Metabolomic, transcriptomic and genetic integrative analysis reveals important roles of adenosine diphosphate in haemostasis and platelet activation in non-small-cell lung cancer. Mol oncol. 2019;13(11):2406-2421. doi:10.1002/1878-0261.12568

42. Fu H, Gao H, Qi X, et al. Aldolase A promotes proliferation and G1/S transition via the EGFR/MAPK pathway in non-small cell lung cancer. Cancer Commun. 2018;38(1):18. doi:10.1186/s40880-018-0290-3

43. Zheng G, Shen Z, Chen H, et al. Metapristone suppresses non-small cell lung cancer proliferation and metastasis via modulating RAS/ RAF/MEK/MAPK signaling pathway. Biomed Pharmacother. 2017;90:437-445. doi:10.1016/j.biopha.2017.03.091

44. Bol GM, Xie M, Raman V. DDX3, a potential target for cancer treatment. Mol Cancer. 2015;14:188.

45. Pelletier J, Thomas G, Volarevic S. Ribosome biogenesis in cancer: new players and therapeutic avenues. Nature rev Cancer. 2018;18 (1):51-63. doi:10.1038/nrc.2017.104

46. Engels EA. Inflammation in the development of lung cancer: epidemiological evidence. Expert Rev Anticancer Ther. 2008;8 (4):605-615. doi:10.1586/14737140.8.4.605

47. Hansen TB, Jensen TI, Clausen BH, et al. Natural RNA circles function as efficient microRNA sponges. Nature. 2013;495 (7441):384-388. doi:10.1038/nature11993

48. Ma Z, Qiu X, Wang D, et al. MiR-181a-5p inhibits cell proliferation and migration by targeting Kras in non-small cell lung cancer A549 cells. Acta Biochim Biophys Sin. 2015;47(8):630-638. doi:10.1093/ abbs/gmv054

49. Mo X, Zhang F, Liang H, Liu M, Li H, Xia H. miR-544a promotes the invasion of lung cancer cells by targeting cadherina 1 in vitro. Onco Targets Ther. 2014;7:895-900. doi:10.2147/OTT.S61695 
50. Zhang ZW, Chen JJ, Xia SH, et al. Long intergenic non-protein coding RNA 319 aggravates lung adenocarcinoma carcinogenesis by modulating miR-450b-5p/EZH2. Gene. 2018;650:60-67. doi:10. 1016/j.gene. 2018.01 .096

51. Wei C, Zhang R, Cai Q, et al. MicroRNA-330-3p promotes brain metastasis and epithelial-mesenchymal transition via GRIA3 in non-small cell lung cancer. Aging. 2019;11(17):6734-6761. doi:10. 18632/aging.102201

52. Zhao W, Chen T, Zhao Y. Upregulated lncRNA CASC9 Contributes to Progression of Non-Small Cell Lung Cancer Through Inhibition of miR-335-3p and Activation S100A14 Expression. Onco Targets Ther. 2020;13:6027-6036. doi:10.2147/OTT.S249973
53. Novak CM, Horst EN, Taylor CC, Liu CZ, Mehta G. Fluid shear stress stimulates breast cancer cells to display invasive and chemoresistant phenotypes while upregulating PLAU in a 3D bioreactor. Biotechnol Bioeng. 2019;116(11):3084-3097. doi:10.1002/bit.27119

54. Tripathi SC, Fahrmann JF, Celiktas M, et al. MCAM Mediates Chemoresistance in Small-Cell Lung Cancer via the PI3K/AKT/ SOX2 Signaling Pathway. Cancer Res. 2017;77(16):4414-4425. doi:10.1158/0008-5472.CAN-16-2874

55. Tan AC. Targeting the PI3K/Akt/mTOR pathway in non-small cell lung cancer (NSCLC). Thorac Cancer. 2020;11(3):511-518. doi:10.1111/1759-7714.13328

\section{Publish your work in this journal}

OncoTargets and Therapy is an international, peer-reviewed, open access journal focusing on the pathological basis of all cancers, potential targets for therapy and treatment protocols employed to improve the management of cancer patients. The journal also focuses on the impact of management programs and new therapeutic

Submit your manuscript here: https://www.dovepress.com/oncotargets-and-therapy-journa agents and protocols on patient perspectives such as quality of life, adherence and satisfaction. The manuscript management system is completely online and includes a very quick and fair peer-review system, which is all easy to use. Visit http://www.dovepress.com/ testimonials.php to read real quotes from published authors. 